\title{
A Nucleosome-Free dG-dC-Rich Sequence Element Promotes Constitutive Transcription of the Essential Yeast RIO1 Gene
}

\author{
Michaela Angermayr*, Kerstin Schwerdtfeger ${ }^{a}$ \\ and Wolfhard Bandlow \\ Department Biologie I, Bereich Genetik der \\ Ludwig-Maximilians-Universität München, \\ Maria-Ward-Strasse 1a, D-80638 München, Germany \\ ${ }^{*}$ Corresponding author
}

$R I 01$ is an essential gene that encodes a protein serine kinase and is transcribed constitutively at a very low level. Transcriptional activation of RIO1 dispenses with a canonical TATA box as well as with classical transactivators or specific DNA-binding factors. Instead, a dG-dC-rich sequence element, that is located 40 to $48 \mathrm{bp}$ upstream the single site of mRNA initiation, is essential and presumably constitutes the basal promoter. In addition, we demonstrate here that this promoter element comprises a nucleosome-free gap which is centered at the dG-dC tract and flanked by two positioned nucleosomes. This element is both, necessary and sufficient, for basal transcription initiation at the RIO1 promoter and, thus, constitutes a novel type of core promoter element.

Key words: Chromatin analysis (in vivo footprinting) / Constitutive promoter/Essential gene/Gene shuffling/ Promoter analysis/Protein serine kinase /

Saccharomyces cerevisiae.

RIO1 is an essential gene which codes for a protein serine kinase highly conserved from Archaea to man (Angermayr et al., 2002a; Angermayr and Bandlow, 2002). The biological role of RIO1 and members of the RIO1-like kinase family is largely unknown. In Saccharomyces cerevisiae Rio1p plays an essential role in cell cycle progression, i.e. in the transition from $\mathrm{G} 1$ to $S$ phase and in metaphase, before onset of anaphase. However, there are indications that Rio1p influences 18S rRNA maturation and certain steps in the assembly of the small ribosomal subunit as well (Vanrobays et al., 2002).

RIO1 is transcribed constitutively at an extremely low level (Angermayr and Bandlow, 1997a,b). In contrast to core promoters of regulated genes (e.g. Chen and Struhl,

\footnotetext{
a Present address: Institute of Cell Biology, ETH Zürich, Hönggerberg HPM E48, CH-8093 Zürich, Switzerland
}

1988; Harbury and Struhl, 1989), little is known about the molecular basis of constitutive gene expression, and the DNA sequence elements involved are poorly defined. The RIO1 promoter shares an 869 bp comprising upstream region with the divergently transcribed GCY1 gene. This intergenic region provides an interesting model to study gene-specific regulation. Expression of GCY1 is induced 25 -fold by growth on galactose due to the binding of the galactose-inducible transactivator Gal4p to a single upstream activating sequence $\left(\mathrm{UAS}_{\mathrm{GAL}}\right)$. Although Gal4p acts bidirectionally and over long distances, expression of RIO1 is not influenced by this specific transactivator. Previous work (Angermayr and Bandlow, 1997a, 2003) has shown that the core promoter of RIO1 allows assembly of a basal transcription complex that differs from the one recruited to the TATA box of GCY1 and that does not respond to Gal4p. Here, we studied the molecular basis of the constitutive activation of the RIO1 promoter.

Apart from the remote $U_{A S}$ (in front of GCY1), we found several remarkable nucleotide sequences in the region 5'-upstream of the RIO1 gene, i.e. two closely spaced poly(dA:dT) tracts, as well as each a GC- and an AT-rich sequence block; however, a canonic TATA motif was absent. In a first attempt to define the minimal promoter required for constitutive transcription of $R I O 1$, we truncated the intergenic region of GCY1 and RIO1 stepwise from the GCY1 side. Since RIO1 is essential, we could not create mutations that yield a non-functional promoter into the genuine genomic context. So we decided to use plasmid-based lacZ reporter constructs. In previous studies on the GCY1 promoter, initiating a transcript divergent to RIO1 (Angermayr and Bandlow, 2003), as well as on the PFY1-promoter (Angermayr et al., 2003), we had observed that lac $Z$ expression data obtained with plasmid-borne wild-type and mutant constructs fully parallelled transcript levels of the same mutant promoters in the genuine genomic background (however, GCY1 and PFY1 are non-essential genes in contrast to RIO1). We ligated the respective shortened promoter elements to pYLZ2 as a lacZ expression plasmid (Hermann et al., 1992) and determined $\beta$-galactosidase activities (Angermayr and Bandlow, 1997b; Figure 1A). The promoter of RIO1 is extremely weak (Angermayr and Bandlow, 1997a). On the basis of the transcriptome analysis, it has been calculated that the concentration of RIO1-mRNA in the steady-state is about one molecule per cell or even less (Holstege et al., 1998). In the wild type, RIO1-mRNA 
A

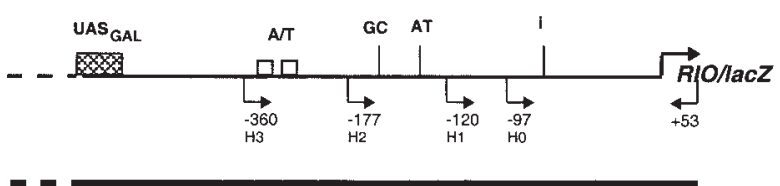

B-galactosidase activities (U/mg)

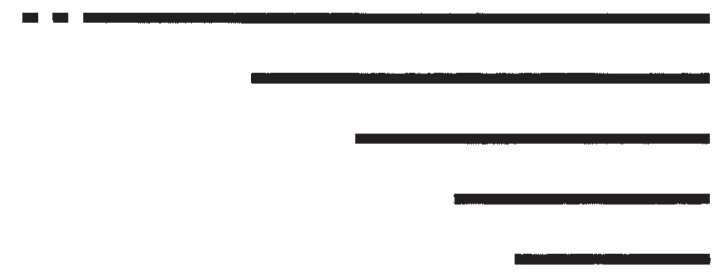

B
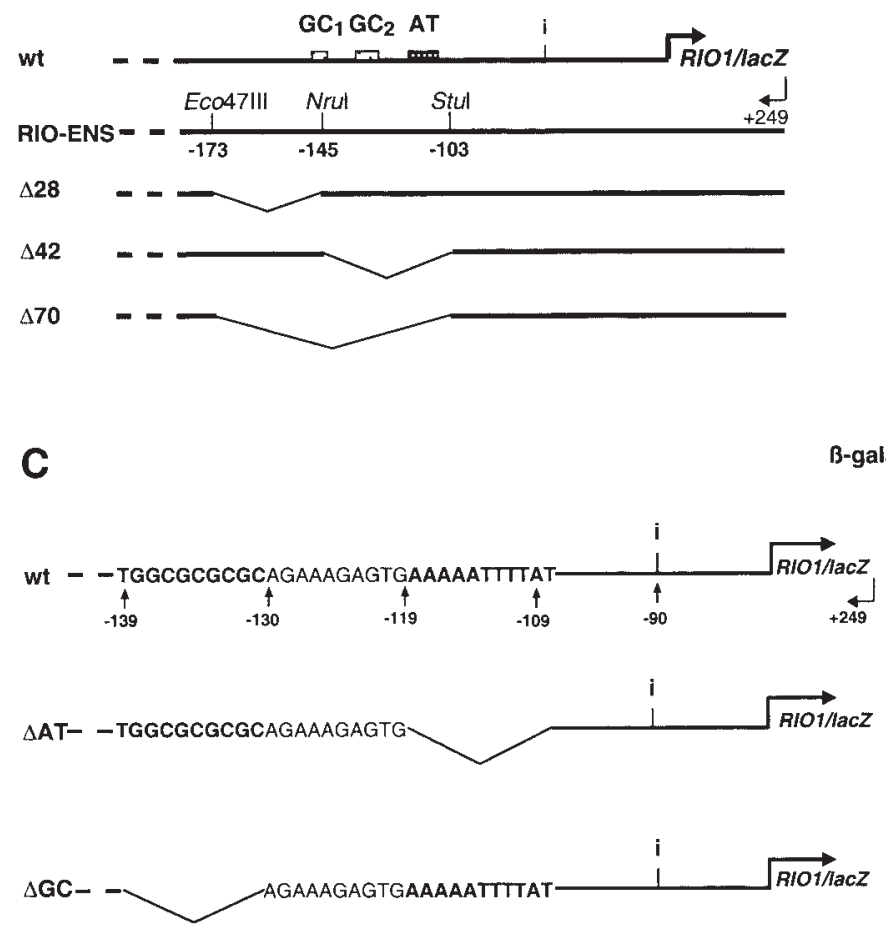

Fig. $1 \beta$-Galactosidase Activities of Various Mutants of the RIO1 Promoter.

(A) Schematic drawing of the promoter of the RIO1 gene. Forward primers for the truncations and their positions are indicated. The right column gives $\beta$-galactosidase reporter activities (Angermayr and Bandlow, 1997a,b) of the yeast reporter plasmid pYLZ2 (Hermann et al., 1992). (B) Introduction of restriction sites into the presumptive core promoter of RIO1 and measurement of the respective reporter activities. GC- and AT-rich sequences are indicated. (C) Deletion of short sequence blocks and respective $\beta$-galactosidase reporter activities. Reporter activities differ between the experiments shown in $(A)$ and $(B, C)$ due to different constructs using different reverse primers (the construct in $\mathrm{B}$ and $\mathrm{C}$ contains a longer 5'-coding part of RIO1 than in panel A).

escapes detection by Northern blotting, and expression of RIO1 promoter-lacZ fusions from low copy plasmids hardly differs from background. For this reason, all reporter constructs were expressed from the multi-copy plasmid pYLZ2.

Truncation of the RIO1 promoter by means of the forward primers $\mathrm{H} 2, \mathrm{H} 3$ or $\mathrm{H} 4$, reveals that reporter expression stays at a relatively constant level (Figure 1A) indicating that no important cis element lies within this region. This observation furthermore shows that the two (dA:dT) blocks, lying between primers $\mathrm{H} 2$ and $\mathrm{H} 3$, have no significant bearing on the expression of RIO1. Transcription of RIO1 is eliminated when the region between positions -177 and -120 is deleted (primer $\mathrm{H} 1$ ). Thus, the cis-element essential for expression of RIO1 lies within a 57 bp interval between positions -177 and -120 . Transcriptional activation of unregulated genes frequently employs 'general regulatory factors'. These are highly abundant and frequently assist to recruit other factors and/or play an architectural role. They are easily detected both, by their strong retention signal in electrophoretic mobility shift assays (e.g. McLean et al., 1995; Anger- 
mayr and Bandlow, 1997b; Angermayr et al., 2003) and/or in silico by their well-defined DNA-binding motifs. However, we did not find any indications for a specific transactivator protein binding to the promoter region of RIO1. The apparent absence of binding of a classical transactivator or general transcription factor argues that synthesis of RIO1 mRNA exclusively is basal transcription.

To narrow down the promoter requirements for expression of RIO1, we introduced restriction sites into the above determined region by in vitro mutagenesis taking care of minimal sequence alterations. These constructs differed from those shown in Figure $1 \mathrm{~A}$ in that a different reverse primer was used which annealed at nucleotide positions +249 to +231 , whereas in the constructs displayed in Figure $1 \mathrm{~A}$ the reverse primer was complementary to positions +53 to +35 . The longer construct yielded higher expression as the respective fusion protein proved to be proteolytically more stable. We inserted an Eco47III site at the 5'-end (position -173), and a Stul site at the 3'-end of the essential promoter of RIO1 (position -103). In addition, we introduced an Nrul site at position -145 . By these means, we were able to delete the whole essential promoter region on the one hand, and on the other hand eliminate either half of these sequences (Figure 1B). Neither the restriction sites introduced, nor the deletion of the most upstream $28 \mathrm{bp}$ of the $70 \mathrm{bp}$ comprising segment in question influenced promoter activities of RIO1. However, when we deleted the $42 \mathrm{bp}$ $\mathrm{Nrul} / \mathrm{Stul}$ fragment, promoter activity was completely impaired. The same was the case for deletion of the $70 \mathrm{bp}$ total promoter region.

The 42 bp segment essential for expression of R/O1 excels by containing a block of nucleotides with extremely high GC content followed by an AT-rich stretch. It is located 13 to $55 \mathrm{bp} 5^{\prime}$ from the single transcriptional initiation site at position -90 (Angermayr and Bandlow, 1997b). In yeast, transcription usually starts about 40 to 120 bp downstream the core promoter. Therefore, it seems plausible that this region comprises the core promoter of RIO1. To test this possibility, we eliminated either the GC-rich or the AT-rich block by site specific in vitro mutagenesis (Figure 1C). The same reverse primer was used as described for Figure 1B. Deletion of the AT-rich sequences had no effect on RIO1/lacZ expression. However, deletion of the GC-block destroyed promoter activity completely. Thus, the eight bp-comprising GC block constitutes an essential promoter element, presumably the basal promoter specifically required for transcription of $R I O 1$.

In order to obtain evidence that the GC-rich sequence block has functional importance for the expression of RIO1 in vivo, we performed a 'gene shuffling' experiment. A strain disrupted for RIO1 was rescued by a wild-type copy of the gene on a CEN-based URA3 plasmid (YMA51; Angermayr et al., 2002a). In addition, it harboured a second (LEU2)-plasmid containing either a wild-type copy, too (control), or a deletion of the GC- or
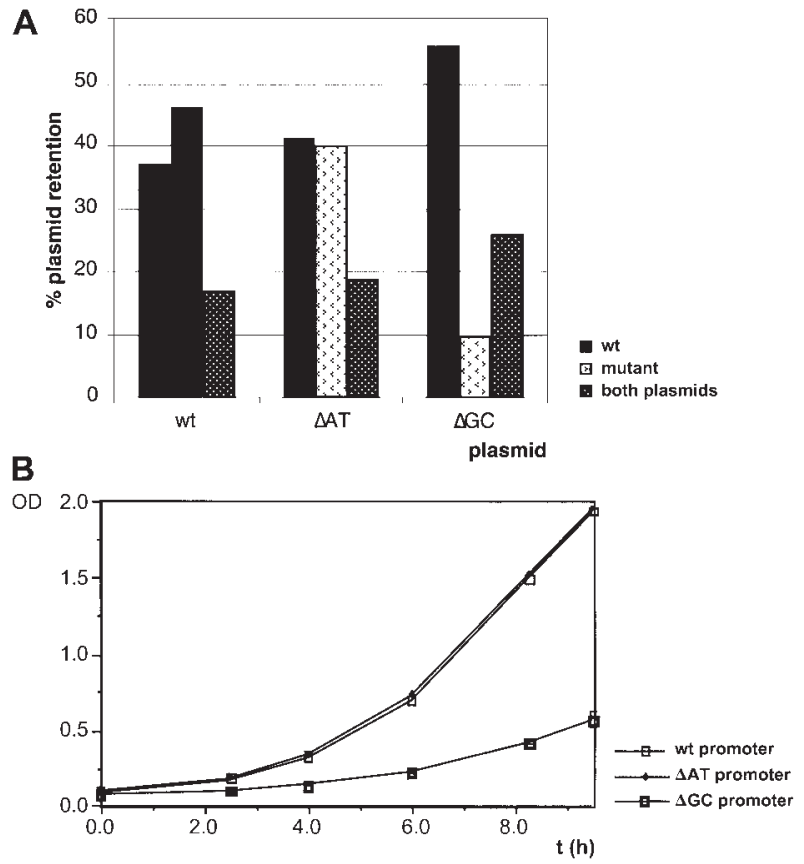

Fig. 2 Plasmid Segregation Test under Non-Selective Conditions.

A strain deleted for RIO1 was rescued by a RIO1 wild-type copy on a CEN-based URA3 plasmid (YMA51) and simultaneously contained either a wild-type RIO1 (control) or $\triangle \mathrm{GC}$ or $\triangle \mathrm{AT}$ promoter deletion mutant on a LEU2 CEN plasmid. The two plasmids were allowed to segregate for 30 generations in complete medium and then tested for the markers retained (panel A). Panel (B) displays growth curves of YMA52 (wild-type control) and one of the segregants containing either the $\triangle \mathrm{GC}$ or the $\triangle \mathrm{AT}$ mutation. Identical results were obtained with two other segregants each.

AT-rich blocks. Subsequent segregation was performed in the presence of 5-fluoro-orotate selecting against the presence of the URA3 plasmid, under semi-selective or under non-selective conditions with similar results. Figure $2 \mathrm{~A}$ shows the data obtained under non-selective conditions which are most meaningful as they reflect the importance of the respective plasmid for sustaining cell viability. In the control, either of the two wild-type RIO1 copies are lost with comparable probability, although loss of the URA3 plasmid generally is slightly more frequent (compare with Angermayr et al., 2002a). The plasmid carrying the deletion of the AT block is retained at similar frequency as wild type, and its retention as the sole RIO1 plasmid displays no phenotype (not shown). The plasmid harbouring the deletion of the GC block is retained in a few rare occasions as the sole RIO1 copy, but the phenotype of these cells is very sick and closely resembles cells deprived of Rio1p (after shift from galactose to glucose medium of a RIO1 deleted strain rescued by $R I O 1$ under guidance of the GAL10 promoter): giant wrinkled cells, frequently arrested in G1 (not shown here, but compare with Angermayr et al., 2002a). In addition, these cells display an extreme slow growth phenotype (Figure 2B) and enter stationary phase at a very low titre 
$\left(2 \times 10^{7}\right)$. These observations demonstrate the importance of the GC-rich sequence block for the expression of Rio1p also in vivo, although its deletion allows survival at the border of viability. Similarly, residual transcription at a very low level has been observed with other genes after deletion of their TATA core promoter (e.g. Angermayr and Bandlow, 1997a). In addition, yeast cells frequently harbour more than one copy of CEN-plasmids ( $1-3$ copies per cell). Therefore, more than one copy of RIO1 under the mutant promoter likely sustains viability.

Next we examined the chromatin structure at the RIO1 promoter in the genomic context. Generally, unregulated transcription reflects a static situation. It is assumed that constitutive promoters permanently are in an active state and kept free of nucleosomes and, thus, accessible to the basal transcription machinery. In chromatin, normal B-helical DNA is wrapped around histone octamers which usually are arranged in regular nucleosomal structures (Workman and Kingston, 1998; Becker and Hörz, 2002). To meet the specific requirements for transcriptional gene activation, core promoter sequences of constitutive promoters are presumed to lie in a nucleosomefree gap of the chromatin. Core promoters of actively transcribed genes have been found to be free of nucleosomes (e.g. Fascher et al., 1990; Piña et al., 1990). Revealingly, it has been observed that experimentally induced depletion of yeast cells of histone $\mathrm{H} 4$ leads to maximal activation of several genes even in the absence of activator (Han and Grunstein, 1988; Durrin et al., 1992). This shows that in these instances the major task of the respective transcription factor(s) is to affect or mediate removal of repressing nucleosome(s) and that basal transcription can dispense with classical transactivators as long as the basal promoter provides a freely accessible platform for the assembly of the basal transcription complex.

The absence of binding sites for architectural proteins or classical transcription factors led us to presume that the DNA sequence or structure at the basal promoter could directly be responsible for providing the prerequisites for the assembly of the basal transcription complex. Therefore, we examined whether the promoter in fact is free of nucleosomes. We analysed the chromatin structure at the RIO1 promoter by DNasel or MNase digestion (Figure 3). In 'naked' DNA, the entire promoter region is readily sensitive to digestion by either nuclease indicating that it does not contain sequence-determined constraints hindering nucleolytic degradation. Also in native chromatin, the core promoter is freely accessible to nucleolytic attack by either nuclease and, consequently, is free of nucleosomes. Apparently, no additional DNAbinding proteins are necessary to remove nucleosomes in order to enable access of the basal transcription complex to the core promoter. The nucleosome-free gap spans about $130 \mathrm{bp}$ of promoter DNA. It is centered at the GC-rich core promoter and comprises the transcriptional initiation site as well.

Upstream of the hypersensitive site, a region follows to the 5'-side of the promoter that is not well cleaved by either nuclease in 'naked' DNA. In chromatin, however, the

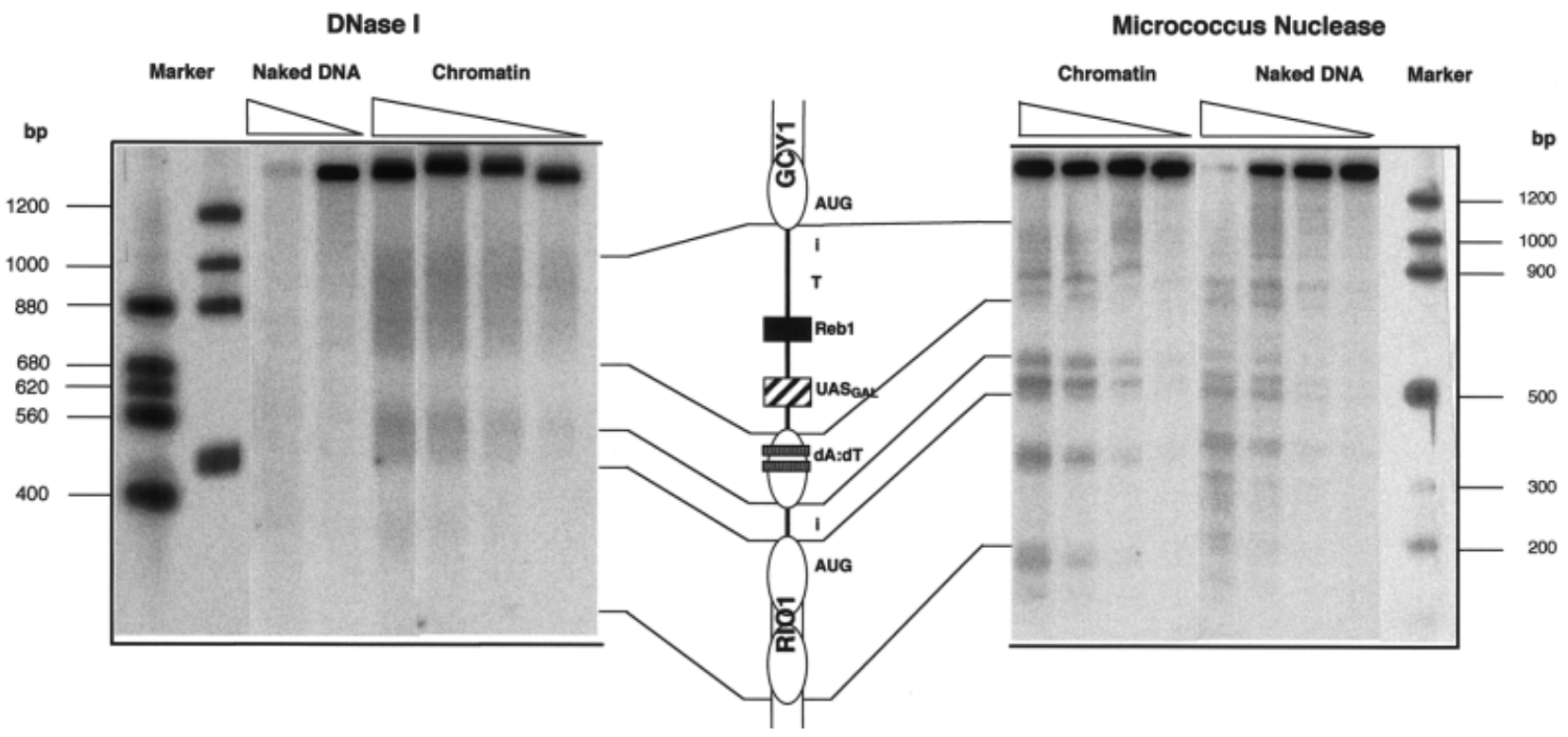

Fig. 3 Chromatin Structure Analysis of Promoter and Coding Region of RIO1 in the Genomic Context.

Native chromatin of the wild type was digested with increasing concentrations of DNasel or micrococcal nuclease (Thoma, 1996), and DNA then deproteinated, treated with restriction endonuclease to create defined ends, electrophoresed and detected by indirect endlabelling (see Angermayr et al., 2002b and Angermayr and Bandlow, 2003 for details). DNA fragment length standards are given at the margins. The results are illustrated by the corresponding schematic drawing in the centre. Ellipses symbolise nucleosome-protected DNA, wide open bars indicate coding, narrow bars depict non-coding hypersensitive regions. The TATA box (T), the Reb1p-binding site and the Gal4p box of the adjacent GCY1 promoter as well as the position of two dA:dT 13-meric blocks, the transcriptional initiation sites (i) and the AUG translational initiation sites are indicated. 
nuclease-insensitive region is much wider and significantly extends in both 5' and 3' direction relative to 'naked' DNA, indicating that in chromatin it is protected by a nucleosome veiling about $150 \mathrm{bp}$ of DNA. This nucleosome is centered at the $21 \mathrm{bp}$ spacer between two 13-meric (dA: $d T$ ) blocks and, as the boundaries of the protected sequence are relatively sharp, appears to be positioned. Packaging of dA:dT sequences into nucleosomes is controversially discussed. It has been reported that DNA containing $\mathrm{dA}: \mathrm{dT}$ blocks is incompatible with wrapping around nucleosomes due to their rigid structure (Struhl, 1985; lyer and Struhl, 1995); however, arrangement of short tracts of homooligomeric dA:dT sequences in a nucleosomal structure is perfectly possible (Kunkel and Martinson, 1981). Thus, it appears that the $21 \mathrm{bp}$ of spacer DNA between the two homooligomeric $\mathrm{dA}: \mathrm{dT}$ blocks helps to position the single upstream nucleosome (see schematic drawing in Figure 3).

The two nucleosomes flanking the gap on the 3'-side protect 150 bp of DNA each. The boundaries are sharp, and the spacer is narrow so that they seem to be positioned as well. Thus, the core promoter lies in a gap between two positioned nucleosomes.

Several principles have been found to govern preferred positioning of nucleosomes and creation of nucleosome-free gaps. Sequence-dependent binding of two nucleosomes to two adjacent sites of prebent DNA may lead to translational positioning of nucleosomes and allow access of transcriptional activators to the extended linker between them. The linker DNA may, however, be too short to allow the binding of an additional nucleosome. Such an arrangement is the basis of accessibility of linker DNA to specific transactivators in several promoters (Fascher et al., 1990; Piña et al., 1990; Travers, 1990; Archer et al., 1991; Straka and Hörz, 1991; Angermayr et al., 2002b). As an alternative, poly(dA:dT) sequences have been observed to deviate from the $B$ conformation of DNA and to be incompatible with packaging into nucleosomes due to their rigid DNA structure (Struhl, 1985; Chen et al., 1987; Tanaka et al., 1992; lyer and Struhl, 1995). However, GC-rich sequences may display similar incompatibilities (lyer and Struhl, 1995; Angermayr and Bandlow, 2003).

By DNA permutation analysis (Zinkel and Crothers, 1987), we failed to detect any indication of extensive structural DNA deformation or bending of the GC block (not shown) which could be the cause for nucleosome exclusion. However, it has been reported that in dG-dCrich sequences the DNA structure is distorted in a way that the minor groove is wider than in random DNA (Yoon et al., 1988), but presumably the deformation is too small to be detected by permutation analysis. Since on the one hand, the TATA-binding protein (TBP) binds to the minor groove (Lee et al., 1991; Starr and Hawley, 1991) and bends it towards the major furrow (Horikoshi et al., 1992; Starr et al.,1995) and since on the other hand, prebending of a core promoter sequence enhances the affinity of the basal transcription complex to DNA (Parvin et al.,
1995), an enlarged minor groove could facilitate recruitment of transcription factor IID (TFIID) to dG-dC sequences and allow transcription initiation even in the absence of transactivators. dG-dC-rich sequences have indeed been found to allow assembly of TFIID and to promote transcription in vitro (Singer et al., 1990), and experimental evidence suggests that the TATA-binding protein-associated factor (TAF) composition of this TFIID differs from the one binding to the TATA box of the adjacent Gal4p-controlled GCY1 promoter due to the difference in the structures of the TATA-containing and the GCrich core promoters (Angermayr and Bandlow, 1997a). However, creation of a consensus TATA box in front of RIO1 from the naturally occurring sequence TATAGA by a single base pair exchange increased expression about threefold and rendered it susceptible to induction by galactose (about one hundred-fold; Angermayr and Bandlow, 1997a). This finding demonstrates a principal difference of the two types of core promoters in their responsiveness to regulatory factors and indicates that the type of core promoter is responsible for the low constitutive transcription of RIO1 and for the inability of the basal transcription machinery to interact with the adjacent Gal4p site.

The deviation of prebent dG-dC-rich DNA from the normal B-conformation could also be the cause for nucleosome exclusion. On the other hand, we have shown that the GC-rich promoter element is flanked by two positioned nucleosomes. However, the two 5'-upstream dA:dT blocks probably play only an accessory role in positioning the upstream nucleosome, as their deletion has no effect on Rio1/lacZ expression (Figure 1A). The intervening gap (130 bp) may be too narrow to accommodate an additional nucleosome (cf. Fascher et al., 1990; Angermayr et al. , 2002b, as examples) providing an alternative or additional reason for the existence of the nucleosome-free gap.

Thus, two minimal parameters determine the strength of basal transcription in the absence of transcription factors: (i) accessibility of the core promoter to the basal transcription complex in a nucleosome-free gap of the chromatin and (ii) the structural deformation of core promoter DNA in order to promote binding of TFIID. In line with this conclusion, on the basis of extensive mutational analysis of the basal promoter for Gcn4p-independent transcription of the HIS3 gene it has been postulated that structural rather than sequence peculiarities of the core promoter DNA are responsible for promoter recognition and basal transcription initiation in vivo and in vitro (Mahadevan and Struhl, 1990).

\section{Acknowledgement}

This work was supported by the Deutsche Forschungsgemeinschaft within Sonderforschungsbereich 190, TP B6. 


\section{References}

Angermayr, M., and Bandlow, W. (1997a). The type of basal promoter determines the regulated or constitutive mode of transcription in the common control region of the yeast gene pair GCY1/RIO1. J. Biol. Chem. 272, 31630-31635.

Angermayr, M., and Bandlow, W. (1997b). The general regulatory factor Reb1p controls basal, but not Gal4p-mediated, transcription of the GCY1 gene in yeast. Mol. Gen. Genet. 256 , $682-689$.

Angermayr, M., and Bandlow, W. (2002). RIO1, an extraordinary novel protein kinase. FEBS Lett. 524, 31-36.

Angermayr, M., and Bandlow, W. (2003). Permanent nucleosome exclusion from the Gal4p-inducible yeast GCY1 promoter. J. Biol. Chem. 278, 11026-11031.

Angermayr, M., Roidl, A., and Bandlow, W. (2002a). Yeast Rio1p is the founding member of a novel subfamily of protein serine kinases involved in the control of cell cycle progression. Mol. Microbiol. 44, 309-324.

Angermayr, M., Oechsner, U., Gregor, K., Schroth, G.P., and Bandlow, W. (2002b). Transcription initiation in vivo without classical transactivators: DNA kinks flanking the core promoter of the housekeeping yeast adenylate kinase gene, $A K Y 2$, position nucleosomes and constitutively activate transcription. Nucleic Acids Res. 30, 4199-4207.

Angermayr, M., Oechsner, U., and Bandlow, W. (2003). Reb1pdependent DNA bending effects nucleosome positioning and constitutive transcription at the yeast profilin promoter J. Biol. Chem. 278, 17918-17926.

Archer, T.K., Cordingley, M.G., Wolford, R.G., and Hager, G.L. (1991). Transcription factor access is mediated by accurately positioned nucleosomes on the mouse mammary tumor virus promoter. Mol. Cell. Biol. 11, 688-698.

Becker, P., and Hörz, W. (2002). ATP-dependent nucleosome remodeling. Annu. Rev. Biochem. 71, 247-273.

Chen, W., and Struhl, K. (1988). Saturation mutagenesis of a yeast his3 'TATA element': genetic evidence for a specific TATA-binding protein. Proc. Natl. Acad. Sci. USA 85, 2691 2695.

Chen, W., Tabor, S., and Struhl, K. (1987). Distinguishing between mechanisms of eukaryotic transcriptional activation with bacteriophage T7 RNA polymerase. Cell 50, 1047-1055.

Durrin, L.K., Mann, R.K., and Grunstein, M. (1992). Nucleosome loss activates CUP1 and HIS3 promoters to fully induced levels in the yeast Saccharomyces cerevisiae. Mol. Cell. Biol. 12, $1621-1629$.

Fascher, K.D., Schmitz, J., and Hörz, W. (1990). Role of trans-activating proteins in the generation of active chromatin at the PHO5 promoter in S. cerevisiae. EMBO J. 9, 2523-2528.

Han, M., and Grunstein, M. (1988). Nucleosome loss activates yeast downstream promoters in vivo. Cell 55, 1137-1145.

Harbury, P.A., and Struhl, K. (1989). Functional distinctions between yeast TATA elements. Mol. Cell. Biol. 9, 5298-5304.

Hermann, H., Häcker, U., Bandlow, W., and Magdolen, V. (1992). pYLZ vectors: Saccharomyces cerevisiae/Escherichia coli shuttle plasmids to analyze yeast promoters. Gene 119, $137-141$.

Holstege, F.C., Jennings, E.G., Wyrick, J.J., Lee, T.L., Hengartner, C.J., Green, M.R., Golub, T.R., Lander, E.S., and Young, R.A. (1998). Dissecting the regulatory circuity of a eukaryotic genome. Cell 95, 717-728.

Horikoshi, M., Bertuccioli, C., Takada, R., Wang, J., Yamamoto, T., and Roeder, R.G. (1992). Transcription factor TFIID induces
DNA bending upon binding to the TATA element. Proc. Natl. Acad. Sci. USA 89, 1060-1064.

Iyer, V., and Struhl, K. (1995). Poly(dA:dT), a ubiquitous promoter element that stimulates transcription via its intrinsic DNA structure. EMBO J. 14, 2570-2579.

Kunkel, G.R., and Martinson, H.G. (1981). Nucleosomes will not form on double-stranded RNA or over poly(dA).poly(dT) tracts in recombinant DNA. Nucleic Acids Res. 9, 6869-6888.

Lee, D.K., Horikoshi, M., and Roeder, R.G. (1991). Interaction of TFIID in the minor groove of the TATA element. Cell 67 , 1241 - 1250 .

Mahadevan, S., and Struhl, K. (1990). T., an unusual promoter element required for constitutive transcription of the yeast HIS3 gene. Mol. Cell. Biol. 10, 4447-4455.

McLean, M., Hubberstey, A.V., Bouman, D.J., Pece, N., Mastrangelo, P., and Wildeman, A.G. (1995). Organization of the Saccharomyces cerevisiae actin gene UAS: functional significance of reiterated REB1 binding sites and AT-rich elements. Mol. Microbiol. 18, 605-614.

Parvin, J.D., McCormick, R.J., Sharp, P.A., and Fisher, D.E. (1995). Prebending of a promoter sequence enhances affinity for the TATA-binding factor. Nature 373, 724-727.

Piña, B., Barettino, D., Truss, M., and Beato, M. (1990). Structural features of a regulatory nucleosome. J. Mol. Biol. 216, 975-990.

Singer, V.L., Wobbe, C.R., and Struhl, K. (1990). A wide variety of DNA sequences can functionally replace a yeast TATA element for transcriptional activation. Genes Dev. 4, 636-645.

Starr, D.B., and Hawley, D.K. (1991). TFIID binds in the minor groove of the TATA box. Cell 67, 1231-1240.

Starr, D.B., Hoopes, B.C., and Hawley, D.K. (1995). DNA bending is an important component of site-specific recognition by the TATA binding protein. J. Mol. Biol. 250, 434-446.

Straka, C., and Hörz, W. (1991). A functional role for nucleosomes in the repression of a yeast promoter. EMBO J. 10, $361-368$.

Struhl, K. (1985). Naturally occurring poly(dA-dT) sequences are upstream promoter elements for constitutive transcription in yeast. Proc. Natl. Acad. Sci. USA 84, 8419-8423.

Tanaka, S., Zatchej, M., and Thoma, F. (1992). Artificial nucleosome positioning sequences tested in yeast minichromosomes: a strong rotational setting is not sufficient to position nucleosomes in vivo. EMBO J. 11, 1187-1193.

Thoma, F. (1996). Mapping of nucleosome positions. Methods Enzmol. 274, $197-214$.

Travers, A.A. (1990). Why bend DNA? Cell 60, 177-180.

Vanrobays, E., Gleizes, E.-P., Bousquet-Antonelli, C., NoaillacDepeyre, J., Caizergues-Ferrer, M., and Gélugne, J.-P. (2001). Processing of $20 \mathrm{~S}$ pre-rRNA to $18 \mathrm{~S}$ ribosomal RNA in yeast requires Rrp10p, an essential non-ribosomal cytoplasmic protein. EMBO J. 20, 4204-4213.

Workman, J.L., and Kingston, R.E. (1998). Multiple functions of nucleosomes and regulatory factors in transcription. Trends Biochem. Sci. 18, 90-95.

Yoon, C., Prive, G.G., Goodsell, D.S., and Dickerson, R.E. (1988). Structure of an alternating B-DNA helix and its relationship to A-tract DNA. Proc. Natl. Acad. Sci. USA 85, $6332-6336$.

Zinkel, S.S., and Crothers, D.M. (1987). Catabolite activator protein-induced DNA bending in transcription initiation. Nature $382,178-181$.

Received January 29, 2003; accepted May 16, 2003 